\section{OAriginal Communiations.}

\section{ON THE USE OF THE THERMOMETER AS A GUIDE IN THE DIAGNOSIS OF PYREXIAL DISEASES.}

By F. W. Gibson, B.A., M.D.London, late HouseSurgeon of the Taunton and Somerset Hospital, Taunton.

THE object of the following communication is to make the value of the thermometer, as an aid in the study of pyrexial diseases, which is recognised by comparatively few British practitioners, more generally known. Any historical details regarding the history of the progress of this branch of medical science would be out of place here. Those, therefore, who wish for information on this subject, I refer to the chapter on the "Range of Temperature in Disease", to be found in the first volume of Dr. Aitken's most excellent treatise on medicine. Suffice it to say that, although the origin of the employment of the thermometer as an aid in the diagnosis of pyrexial diseases dates back more than a hundred years, yet, until Professor Wunderlich of Vienna published the results of his elaborate researches in 1858, the real importance of daily records of the temperature of the body in such diseases was almost unknown. Drs. Parkes, Jenner, Ringer, and others, are labouring in the same field in this country; and the day is not, I think, very far distant when the physician will consider the thermometer not less indispensable to him than the stethoscope or microscope, and when the surgeon will not neglect the observation of the temperature, since Billroth has brought forward sufficient proofs of its great importance as an assistance in the diagnosis of surgical diseases.

Fluctuations of Temperature vithin the Limits of Health. The normal temperature in the axilla, the only locality in which the temperature is now habitually taken, is $93.5 \mathrm{deg}$; ; but in certain circumstances it may vary a degree or two above or below this point. These variations are, however, not persistent. In a few hours-at most, twenty-four-the temperature becomes normal. The important deduction from this fact is, that a persistent rise above 99.5 deg., or depression below $97.3 \mathrm{deg}$., is a sure sign of disease.

Exposure of the body to the air, even in warm weather, lowers the temperature considerably. I have frequently observed a depression of the temperature, continuing for some hours, after slight operations in which there was no notable shock or hæmorrhage; e. g., a boy, aged 11, admitted for phimosis and gonorrhœea, had a temperature of 100.4 deg. immediately before the performance of the operation of circumcision. For two hours after the operation, the temperature remained below the normal height, although he was in bed, carefully covered up.

The temperature is lowered after a full meal and the use of alcohol. It rises as digestion advances. Ingestion of improper articles of diet in typhoid (and other fevers?) may cause a remarkable rise in the temperature, as is shewn by the following case. A man, aged 21, in the third week of typhoid fever, becoming convalescent, with a temperature nearly normal, ate, at two in the afternoon, a considerable quantity of heavy currant-cake. At eight in the evening, his temperature had risen to $105.6 \mathrm{deg}$.

The temperature is lowered by sleep.

Correlation of the Pulse and Temperature. The rule, "that an increase of temperature of one degree above 980 corresponds with an increase of ten beats of s',e pulse per minute," though true in the main, is, as shown by the following cases, subject to many exceptions. In typhoid fever, I have known a pulse of 100 to exist with a temperature of $105.6 \mathrm{deg}$; in jaundice, a pulse of 58 with a temperature of 103 ; in rheumatic fever, a pulse of 80 with a temperature of $103.5 \mathrm{deg}$. ; in peritonitis, a pulse of $80 \mathrm{with}$ a temperature of $102.2 \mathrm{deg}$; in tetanus, a pulse of 72 with a temperature of $104 \mathrm{deg}$; in shock following severe surgical injury, a pulse of 120 with a temperature of $98.5 \mathrm{deg}$; in typhoid fever, a pulse of 120 with a. temperature of $100.4 \mathrm{deg}$.

The temperature is, therefore, a much more certain guide than the pulse, since it is not liable to be affected by nervous debility or excitement. The temperature is, in fact, the only certain means of diagnosis between the frequency of pulse produced by weakness, and that caused by fever; and in those cases, also, in which extreme slowness of the pulse exists as an idiosyncrasy, the employment of the thermometer leads to the discovery of disease which would not otherwise be detected.

The objective temperature of the skin-that is, the sensation conveyed to the applied hand of tho observer-is a most fallacious guide. I have known the skin to be objectively of normal warmth with a temperature of $105 \mathrm{deg}$., and cool with a temperature of $102 \mathrm{deg}$. The use of the thermometer is, therefore, the only reliable method of ascertaining the actual temperature of the body.

As the thermometer has been but little employed hitherto in the diagnosis of pyrexial diseases, a few remarks as to the construction of the instrument, and the proper method of applying it, are necessary before I proceed farther.

The instruments with which I have made most of my observations, I obtained from Mr. Griffin, of Garrick Street, Covent Garden. The scale ranges from 55 to 130 deg., each degree being divided into fifths. Some are covered near the bulb, so as to fit more easily into the armpit; but the advantage thence derived is not great; and $I$ find that the instruments thus made are rather more apt to slip down out of the axilla than those which are straight. The accuracy of the thermometers must be thoroughly established before they are employed, by comparing them with a standard instrument. Dr. Aitken has arranged a maximum self-registering instrument, which does not require to be read in situ, but may be removed and inspected when convenient. Mr. Casella of Hatton Garden supplies this instrument in a box, with a second ordinary thermometer having a bent bulb. Negretti and Zambra also manufacture most excellent instruments. A thermometer, sufficiently accurate for all medical purposes, may be obtained for a moderate sum (seven shillings) of the first named maker (Griffin).

The patient must have been in bed, well covered up, an hour at least before the temperature is taken If he have been lying on his side, he must be turned round, in order that the thermometer may be ap. plied in the axilla which has been next the bed. He must be placed in a medium position between the sicle and back; the bulb of the thermometer passed well up into tive top of the axilla; the arm laid across the chest; and especial care taken that the instru- 
ment be thoroughly surrounded by the soft parts, that no clothes intervene, and that the patient keep his arm in place.

If an approximately accurate observation be all that is desired, the thermometer need not be retained in situ more than five minutes; but it is much better to allow it to remain for ten minutes. The plan which I usually adopt is to note the height to which the mercury has risen at the end of five minutes, and then wait for a minute or so; when, if I find that a rise has taken place, I allow the instrument to remain in three or four minutes longer. The rapidity of the rise of the mercury, the pulse, respirations, and state of the skin, should be simultaneously observed.

The daily thermometric changes, with the variations of the pulse and temperature, may be conveniently recorded by means of a diagram such as that here given.

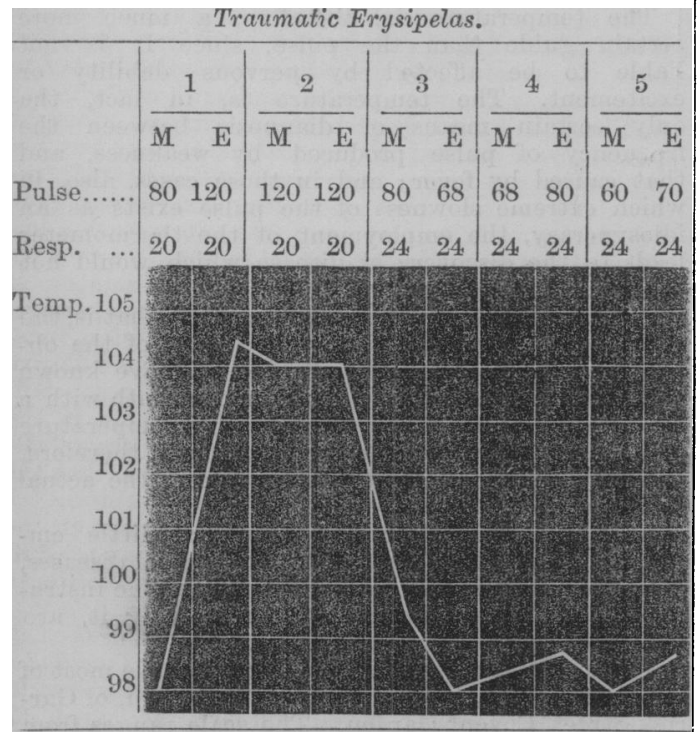

Two daily observations, sufficient in the majority of cases, should be made, as nearly as possible at the same time each day, between the hours of seven and nine in the morning and five and seven in the evening. If more frequent observations be deemed desirable, midnight and noon are considered the most advantageous hours.

Range of Temperature in Disease. The height of the temperature is usually a sure indication of the severity of the fever. If, in a case of typhoid, the temperature in the evening during the second week do not rise above $104 \mathrm{deg}$., the disease may be expected to run a mild course; whereas, if it amount to 105 deg. in the evening or 104 deg. in the morning, the patient is in considerable danger. In traumatic erysipelas, however, the temperature frequently rises with extreme rapidity to a very high degree, withcut the supervention of any alarming symptoms. For example, a man aged 60, under treatment for ulcer of the leg, was infected with erysipelas by a patient in the same ward. The attack began at eight in the morning with a fit of severe rigors ; at nine, his temperature was 104 deg.; it remained high till the morning of the third day, when it had fallen to the normal standard. The disease was superficial, and did not spread above the groin. At no time were there any alarming symptoms. Again : a man aged 22, an epileptic, who had two or three small ulcers on his head, produced by tartar emetic ointment, con- $\overrightarrow{\vec{S}}$ tracted erysipelas. The attack was slight; no danger threatened; nevertheless, his temperature rose to $\frac{}{C}$ $104.6 \mathrm{deg}$. In none of these cases, however, was the $\bar{\sigma}$ high temperature of long continuance.

When, however, the temperature in any felbrile $\frac{\sigma}{\overparen{D}}$ disease approaches $106 \mathrm{deg}$. , the patient is in immi- $\cong$ nent danger. "A temperature of 108 and $109 \mathrm{deg}$. " indicates most surely the near approach of death.; (Wunderlich, who records a case of idiopathic tetanus $\vec{\circ}$ in which, at the moment of death, the mercury stood at $112.5 \mathrm{deg}$.; after death, it rose to $113.8 \mathrm{deg}$.)

The highest temperature which I have observed $O$ occurred in a case of pyæmia, in a woman aged 40 , $\frac{\partial}{3}$ who had her forearm amputated for cancerous disease of the wrist. At two in the afternoon of the ninth day after the operation, she had a se rere at- iv tack of rigors. At half-past three, a temperature of $\longrightarrow$ 103.6 deg. was noted. It rose continuously-a sign in of evil augury in very many cases of pyrexia-until $\frac{\overrightarrow{0}}{6}$ death, which took place thirty-eight hours after the 0 attack of rigors. An hour before death, it had reached 107 deg.

In pneumonia, the height of the temperature gives $\vec{\circ}$ a much surer indication of the presence and amount of pyrexia than either the rate of the pulse and re- $\frac{0}{5}$ spirations, or the physical signs. The latter con- $\stackrel{\bigcirc}{\supset}$ tinue in statu quo, in many cases, long after the tem- $\vec{D}$ perature has fallen to the normal standard. The physician, therefore, who judges from these symptoms alone, will be in danger of falling into the error of unnecessarily prolonging antiphlogistic treatment. On the other hand, the temperature is in some cases very high whilst the physical signs afford $\bar{\partial}$ but slight evidence of the amount of impending disease.

For example, a man, aged 45, with signs of pneu- $\stackrel{\mathbb{Q}}{2}$ monic consolidation extending as far upwards pos- $\vec{F}$ teriorly as the sixth rib, had a respiration rate of 32 윽 per minute, yet his temperature was perfectly normal. A woman, aged 19, having similar signs, with pulse 100 and respirations 32 , had a perfectly normal temperature. A boy, aged 14, in whom the physical signs consisted merely of slight dulness with feeble bronchial respiration at the base, was found to have a temperature of $107 \mathrm{deg}$. How, in the first named cases, could the absence of pyrexia have been dia- 3 gnosed-how, in the last, could the impending se-. verity of the attack (for severe it became) have of been prognosticated-without the aid of the ther- 3 mometer?

Dr. Ringer (Temperature of the Body in Phthisis and Tuberculosis) has proved that there is a continu- N ous elevation of temperature in all cases in which $a$ deposit of tubercle is taking place; that the tem- $\frac{7}{0}$ perature is a more accurate indicator of the amount of disease than either the physical signs or symp- $N$ toms; that, by means of the temperature, we can $\mathrm{N}$ diagnose phthisis long before any physical signs or $\mathrm{W}$ symptoms can be detected; that, by means of the thermometer, we can decide when the deposit of tu- 0 bercle has ceased; and that, by means of that instrument, we can diagnose phthisis from diseases which $\Phi$ more closely resemble it-for example, carcinoma of ? the lung, aortic aneurism, and dilated bronchi. By means of the thermometer, we can diagnose tubercular meningitis from cerebral tumour.

An abscess lying in front of a large artery some- $\stackrel{\mathbb{Q}}{\overparen{D}}$ times so closely resembles aneurism that the diagnosis $\overrightarrow{\mathbb{D}}$ is excessively difficult. In every case of abscess which $\sigma$ I have observed, the temperature has been above the normal standard-often considerably. An ab-? normally high temperature has, in not a few cases, 음 led me to the diagnosis of impending abscess for some hours before fluctuation could be detected. 
The temperature begins to rise at the commencement of the process of suppuration. It rises rapidly until the process is mature, when it begins to fall.

For example, a man, aged 39, was admitted for a severe bruise of his thigh. On the evening of the third day after the accident, his temperature was $103 \mathrm{deg}$. Hardness and intumescence of the soft parts was to be felt near the seat of the injury, but no fluctuation. A poultice was applied. The next evening, his temperature was $105 \mathrm{deg}$. The abscess became matured during the night. The temperature fell gradually next day.

In some cases of severe concussion of the brain, it is of extreme importance to establish with certainty the existence or non-existence of any inflammatory symptoms. Mere nervous shock will in not a few such cases cause the pulse and respirations to be very rapid. The following is a case in point. A boy, aged 14, admitted with severe cerebral concussion, had a pulse of 120 and respirations 36 . The temperature, however, did not rise above $101 \mathrm{deg}$., which proved the absence of any severe inflammatory mischief.

In cases of intestinal hæmorrhage in typhoid fever, the temperature falls for some hours before the blood appears in the stools, the pulse continuing as rapid, or even more rapid, than before the hæmorrhage took place. The thermometer enables the physician to determine the occurrence of the hæmorrhage, and to adopt preventive treatment at once; whereas if, neglecting the use of that instrument, he wait until the blood appears in the motions, much valuable time will have been lost.

$$
\text { [To be continued.] }
$$

\section{ON THE TREATMENT OF STRABISMUS WITHOUT OPERATION.}

By C. Holthouse, Esq., Surgeon to the Westminster Hospital, and to the Surrey Ophthalmic Hospital, etc.

[Continued from page 174. ]

Case II. Double Convergent Strabismus from Paralysis of the External Rectus Muscle of the Left Eye, probably due to slight Capillary Apoplexy. Eliz. W., aged 54, chairwoman, applied to me at the Surrey Ophthalmic Hospital on February 6th, 1860, for a slight convergent strabismus affecting both eyes, diplopia, and much confusion of vision. The movements of the right eye were perfect in every direction, the pupil acted normally, and the vision was good; but the left eye could not be moved outwards beyond a central position between the two canthi; and the upward and outward and downward and outward movements were likewise very limited. In other directions, this eye moved freely, and its pupil and vision were unaffected. On shading each eye alternately, the right or good eye was always more inverted than the left. The diplopia was lateral and homonymous; and the distance between the double images increased as the object gazed at was moved further to the patient's left side.

History. Three weeks previously, while the patient was in church, and during the sermon, her nose began to bleed. She succeeded, however, in stopping the hæmorrhage, but shortly afterwards became affected with a sudden "dizziness", and felt as if she "was going to fall off the seat". On leaving church, she had much pain in the head, which she endeavoured to relieve by tying a handkerchief tightly round her forehead and going to bed. She arose the following morning at her usual hour, and went to work, notwithstanding she felt very unwell, and suffered from much confusion of vision. The above symptoms continued to the time of application. She was or- dered one grain of calomel and one of quinine three times a day.

Feb. 9th. The powders, having griped and purged her freely, were discontinued, and a mixture of three grains of iodide of potassium in an ounce of camphor mixture, to be taken three times a day, was substituted.

Feb. 13th. The paralysis remained without any appreciable diminution, but the patient's health had improved. The medicine was continued.

Feb. 20th. The strabismus and confusion of vision had diminished, and the cornea could be everted to within two and a half lines of the outer canthus. The treatment was continued.

Feb. 24th. The improvement continued; the cornea could be everted to within one line of the outer canthus; and scarcely any perceptible strabismus or confusion of vision remained. The medicine was continued.

The patient ceased to attend after the last note was taken, having considered herself well.

There may be some doubt as to the exact locality of the mischief in the above case ; but less, probably, as to its cause. The preceding epistaxis pointed to congestion and rupture of the capillaries of the nose; and, had the hæmorrhage been encouraged, it might possibly have warded off the paralytic attack ; but, being suppressed before the vessels had sufficiently relieved themselves, the preexisting congestion gave rise to rupture elsewhere; and this extravasation implicated, either at its origin or in some part of its course, the left abducens nerve. The dizziness, of which the patient complained on her first seizure, would not necessarily denote a cerebral lesion, inasmuch as any sudden distortion of one eye will give rise to much confusion of vision and giddiness; but, coupling the latter symptom with the pain in the head and with the absence of paralysis of any of the other ocular muscles, it seems more probable that the extravasation was intracranial than intraorbital.

As regards the influence of the treatment on the favourable result, I am unwilling to speculate. Suffice it that the patient recovered within three weeks of its commencement.

CASE III. Double Convergent Strabismus of the Left Eye to one Line, and of the Right to one Line and a half, from Paralysis of the Left External Rectus Muscle, probably of cerebral Origin. Robert B., aged 35, hricklayer, and healthy looking, applied to me on January 9th, 1866, for a double convergent strabismus affecting the left eye to one line, and the right to one line and a half, when the head was straight; but, left to himself, he kept his head rotated on its axis to the left shoulder, thus making his eyes parallel, and so avoiding diplopia.

The attack came on without obvious cause, and unaccompanied with pain in the head or vertigo. He confessed, however, to having suffered from occasional giddiness and confusion, on stooping down, for the last two or three years.

On testing the movements of each eye separately, those of the right were found to be normal; but the cornea of the left could not be moved beyond the centre of the palpebral aperture. In all other directions, not influenced by the external rectus, the movements of the eye were perfect. The vision of each eye was good, but the accommodation of the left rather less perfect than the right, being in the right eye $\frac{1}{4}$, and in the left $\frac{1}{5}$. After some trials and shifting of the head, he was able to see stereoscopically through the ordinary refracting stereoscope; proving, as will be shown hereafter, that he had the power of keeping the eyes parallel. When first seized with the paralysis, and for some time afterwards, he was much annoyed with the diplopia; but 